\title{
Pre-equilibrium emission and its possible relation to $\alpha$-clustering in nuclei
}

\author{
T. Marchi ${ }^{1}$, F. Gramegna ${ }^{1}$, D. Fabris ${ }^{2}$, M. Degerlier $^{3}$, \\ O.V. Fotina ${ }^{4,5}$, V.L. KravchuK ${ }^{6}$, M. D'Agostino $^{7}$, L. Morelli $^{7}$, \\ S. Appannababu ${ }^{1}$, G. Baiocco ${ }^{7}$, S. Barlini ${ }^{8}$, M. Bini $^{8}$, A. Brondi $^{9}$, \\ M. Bruno ${ }^{7}$, G. Casini ${ }^{8}$, M. Cinausero ${ }^{1}$, N. Gelli $^{8}$, R. Moro ${ }^{9}$,

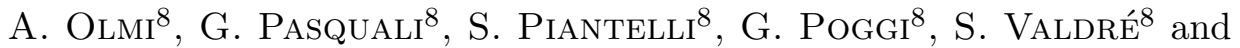
E. VARDACI $^{9}$

${ }^{1}$ INFN-Laboratori Nazionali di Legnaro, Legnaro (PD), Italy

${ }^{2}$ INFN, Sezione di Padova, Padova, Italy

${ }^{3}$ Phys. Dep., Nevsehir Haci Bektas Univ., Nevsehir, Turkey

${ }^{4}$ Phys. Dep., Lomonosov MSU, Moscow, Russia

${ }^{5}$ Skobeltsyn Inst. of Nucl. Phys., Lomonosov MSU, Moscow, Russia

${ }^{6}$ National Research Center "Kurchatov Institute", Moscow, Russia

${ }^{7}$ Phys. Dep., Bologna Univ. and INFN, Sezione di Bologna, Bologna, Italy

${ }^{8}$ Phys. Dep., Firenze Univ. and INFN, Sezione di Firenze, Firenze, Italy

${ }^{9}$ Phys. Dep., Napoli Univ. and INFN, Sezione di Napoli, Napoli, Italy

\begin{abstract}
The study of nuclear states built on clusters bound by valence neutrons in their molecular configurations is a field of large interest. Clustering becomes particularly important at the dripline, where weakly bound systems prevail. For light nuclei, at an excitation energy close to the particle separation value, there are experimental evidences of such structure effects, but this is still not the case for heavier nuclear systems. Several theoretical efforts have been done in the development of pre-formation alpha-clustering models, but there is still a lack of experimental data capable to give a direct feedback. The search of alpha-cluster evidences in medium-mass systems is therefore a new challenge which can give new hints in this field of research.
\end{abstract}


In the past we have studied the reactions 250,192 and $130 \mathrm{MeV}{ }^{16} \mathrm{O}$ $+{ }^{116} \mathrm{Sn}$, observing a significant increase in the fast emitted $\alpha$-particle yield. This effect was ascribed to the presence of pre-formed $\alpha$-clusters in the ${ }^{16} \mathrm{O}$ projectile nucleus. To further investigate these aspects in a model independent way, a new experimental campaign has been performed with the GARFIELD + RCo set up. Two different reaction entrance channels $\left({ }^{16} \mathrm{O}\right.$ and $\left.{ }^{19} \mathrm{~F}\right)$ at the same beam velocity $(16 \mathrm{AMeV})$ have been measured, leading to the same ${ }^{81} \mathrm{Rb}^{*}$ compound nucleus. In this contribution we report on the preliminary light-charged particles emission spectra obtained in coincidence with evaporation residues and their comparison with the results obtained from a Hybrid Exciton Model calculation.

\section{Introduction}

The idea that cluster of nucleons might be pre-formed prior to emission from nuclei has been discussed since many years and was originally proposed by Hafstad and Teller in 1938 [1]. More recently a large interest has been readdressed to the problem of clustering in nuclei, in particular to the study of weakly bound light nuclei at the drip lines, where clustering might be the preferred structural mode [2]. Examining the nuclear binding energies as a function of the mass number, the behavior found shows a systematic trend that is well described by the liquid drop model as due to a shell structure effect. In particular, the specific property of the nucleonnucleon force for which a saturation arises due to the zero coupling of both the spin and the isospin quantum numbers, produces a very strong binding of $\alpha$-particles which can therefore be recognized as a unique cluster subsystem in nuclei. The $\alpha$-particle is the main ingredient in the concept proposed by Ikeda in his diagram [3], where highly clustered states are predicted at excitation energies around the energy threshold for the decomposition into specific cluster channels. In the extended Ikeda diagram, moreover, it is suggested that in neutron-rich systems neutrons may act as valence particles which can be exchanged between the $\alpha$-particle cores, in a similar way to how electrons are exchanged in atomic molecules. In the nuclear case the covalent neutrons stabilize the unstable multi-cluster states, giving rise to nuclear structures which may be described as "nuclear molecules". These concepts are well reproduced in model independent approaches like the Fermionic Molecular Dynamics (FMD) by Feldmeier et al. [4,5] or the Antisymmetrized Molecular Dynamics (AMD) with effective N-N forces by Horiuchi and Kamada-EnYo $[6,7]$. Up to now these structures have been mainly described by theory since the low intensity of exotic beams presently available challenges the 
experimental access to such exotic structures. While waiting for the next generation of radioactive beam facilities like SPES [8], HIE-ISOLDE [9] and SPIRAL2 [10] to be operative, it is of particular interest to search for $\alpha$-clustering effects in non-traditional observables, like those deriving from pre-equilibrium process studies, which may bring new information on the cluster formation process.

\section{Previous measurements}

In a previous campaign the decay of the ${ }^{132} \mathrm{Ce}$ compound nucleus $\left({ }^{16} \mathrm{O}+{ }^{116} \mathrm{Sn}\right)$ has been studied with the aim of identifying the amount of pre-equilibrium emission in asymmetric entrance channel reactions at various incident energies. During this campaign an extra yield was observed for pre-equilibrium $\alpha$-particle emission, which was not reproduced by a Hybrid Exciton Model calculation [11]. This model was using a modified version of the PACE2 code, where the main variation was the introduction of a non-equilibrium stage before the complete thermalization and compound nucleus formation. The relaxation process which occurs during the fusion reaction is firstly accounted for by the exciton model, based on the Griffin prescription [12], in which the description of the angular distribution of the fast emitted particles is still an intricate question [13]. The main parameter to be set is the initial number of excitons $\left(n_{0}=n_{\text {particles }}+n_{\text {holes }}\right)$, that can be estimated from the empirical trend described in the work by N. Cindro et al. [14] and it is mainly related to the projectile properties. In the case of the considered ${ }^{16} \mathrm{O}$ induced reactions this number is $\mathrm{n}_{0}=17=16 \mathrm{p}+1 \mathrm{~h}$. Starting from such initial exciton number, a general good description was obtained by model prediction when compared to double differential cross section proton energy spectra at all the incident energies considered. On the contrary, an enhanced fast $\alpha$-particle production was observed experimentally, especially at the most forward measured angles, which was not accounted for by the calculated distributions. A possible explanation of this enhanced $\alpha$-particle emission might be the effects induced by the $\alpha$-cluster structure in the ${ }^{16} \mathrm{O}$ projectile [15]. This effect has therefore been taken into account in the model introducing the cluster pre-formation probability parameter. The combination of different initial configurations has been considered. In particular, the probability of occurrence of a pattern in which the ${ }^{16} \mathrm{O}$ projectile is supposed to be divided into a ${ }^{12} \mathrm{C}$ core plus an $\alpha$ particle has been considered with respect to the original ${ }^{16} \mathrm{O}$ single-core configuration. This free parameter has to be determined from the comparison with the experimental data. Since a 
quite sizable probability (up to 50\%) of $\alpha$-cluster pre-formation was needed to reproduce the existing data, we concluded that further experimental data and more exclusive observables were needed for a better understanding of the whole process.

\section{The experiment}

The two fusion reactions ${ }^{16} \mathrm{O}+{ }^{65} \mathrm{Cu}$ and ${ }^{19} \mathrm{~F}+{ }^{62} \mathrm{Ni}$ have been studied at $16 \mathrm{AMeV}$ incident energy in order to directly compare their light charged particle emission spectra and yield ratios. The same projectile velocity was chosen since the pre-equilibrium emission is expected to mostly depend on this parameter [16]. In this situation, even with an expected small difference in the evaporative part due to the excitation energy $\left(\mathrm{E}^{*}=209 \mathrm{MeV}\right.$ and $\mathrm{E}^{*}=240 \mathrm{MeV}$ respectively), the fast emission process is predicted to be almost the same for both reactions. Any observed difference and overproduction of fast $\alpha$-particles between the two cases would suggest, in a model independent way, a possible influence of the projectile $\alpha$-structure effect. The experiment has been performed at the Legnaro National Laboratory using the GARFIELD + RCo array, fully equipped with digital electronics [17]. Fully identified light-charged particles have been measured both in single and in coincidence with Evaporation Residues (ERs), detected in the RCo. In the preliminary analysis here reported, only the double-differential proton and alpha energy spectra obtained in the GARFIELD angular range, in coincidence with ERs, have been considered. The two reactions show very similar proton spectra except for a small difference at the most forward angles. This effect can be ascribed to the slightly larger excitation energy of the ${ }^{19} \mathrm{~F}$ induced reaction. A much larger difference is, on the contrary, observed in the case of the $\alpha$-particle emission spectra. The evaporative code (PACE4), which takes into account the difference in the excitation energy of the Compound Nucleus, confirms that the purely statistical emission spectra for the two systems should be very similar. When compared to the data, this calculation supports the idea that a second fast emission source for both systems is present. While the complete data sorting and calibration procedure are carried on, a first evaluation of the expected amount of fast emission in the two cases was performed by means of the Hybrid Exciton Model. The calculation was done starting from an initial exciton number of $\mathrm{n}_{0}=17(16 \mathrm{p}+1 \mathrm{~h})$ in the case of ${ }^{16} \mathrm{O}+{ }^{65} \mathrm{Cu}$. With this configuration the shape of the $\alpha$-particle spectra seems reasonably reproduced, except for an underestimation at the most forward GARFIELD angles. Oppositely, using 


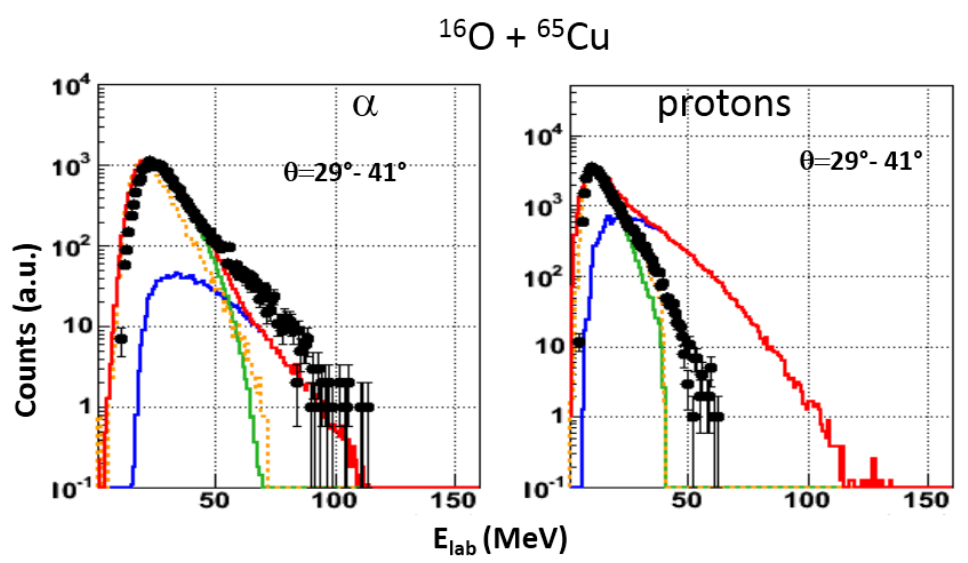

Figure 1: (Colour online) $\alpha$-particle (left panel) and proton (right panel) energy distributions in the laboratory frame for the system ${ }^{16} \mathrm{O}+{ }^{65} \mathrm{Cu}$ : experimental data (black dots) are compared to Compound Nucleus statistical emission from PACE4 (orange dots) and to Hybrid Exciton Model predictions (red line total, green line thermalized emission (PACE2), blue line pre-equiilibrium). The distributions are normalized to the total area.

the same initial parameter, the code strongly overestimates the proton preequilibrium emission. In figure 1 the comparison is shown for $\alpha$-particles and protons in the ${ }^{16} \mathrm{O}+{ }^{65} \mathrm{Cu}$ system at the most forward GARFIELD angular range. Performing the same comparison in the case of the ${ }^{19} \mathrm{~F}+{ }^{62} \mathrm{Ni}$ reaction, where an initial exciton configuration of $n_{0}=20(19 p+1 h)$ was used, a quite similar result is obtained, as shown in 2 . In the ${ }^{19} \mathrm{~F}$ induced reaction case, the experimental fast $\alpha$-particle overproduction is even larger than in the ${ }^{16} \mathrm{O}$ induced reaction, while the fast protons are, again, largely overestimated. A tentative explanation for the observed difference between the $\alpha$-particle decay in the two systems may be the lower energy needed to break up the ${ }^{19} \mathrm{~F}$ nucleus into $\alpha+{ }^{15} \mathrm{~N}(4.01 \mathrm{MeV})$ with respect to the ${ }^{16} \mathrm{O}$ to be divided into $\alpha+{ }^{12} \mathrm{C}(7.2 \mathrm{MeV})$.

By changing the initial configuration parameter (i.e. diminishing the exciton number) the description of the $\alpha$-particle slightly improves, but, on the contrary, this worsen the proton description. Once again, like it was observed in the ${ }^{16} \mathrm{O}+{ }^{116} \mathrm{Sn}$ case, a unique initial parameter in the Hybrid Exciton model is not able to describe both the proton and $\alpha$-particle channels suggesting that some more attention has to be paid to the clustering structure effects. For example in the used calculation no deuteron emission is considered, which may strongly influence the relative proton decay 


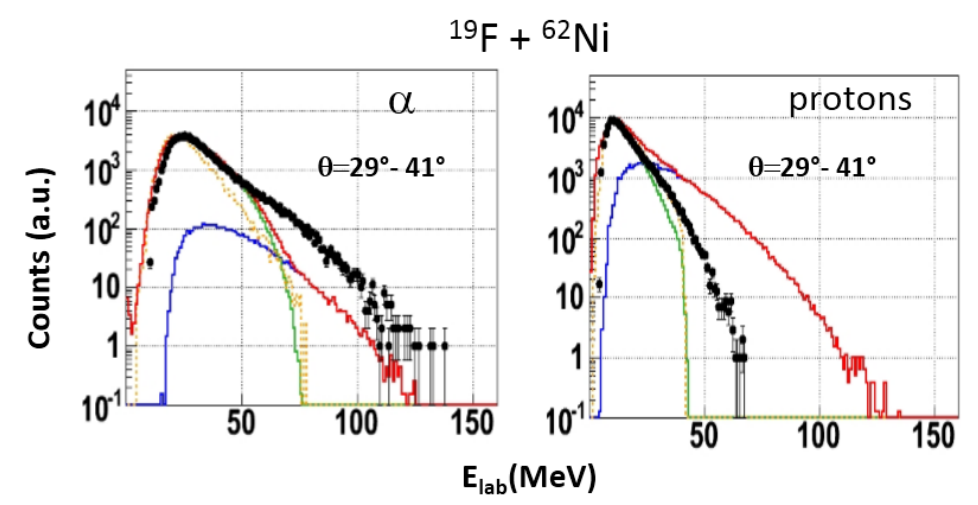

Figure 2: (Colour online) Same as figure 1 for the ${ }^{19} \mathrm{~F}+{ }^{62} \mathrm{Ni}$ system.

probability.

\section{Conclusions}

In order to probe possible $\alpha$-clustering effects in medium-mass nuclei we are studying the secondary particle emission from the $256 \mathrm{MeV}{ }^{16} \mathrm{O}+{ }^{65} \mathrm{Cu}$ and $304 \mathrm{MeV}{ }^{19} \mathrm{~F}+{ }^{62} \mathrm{Ni}$ systems. A difference between the two systems has been underlined in the fast $\alpha$-decay channel and has been ascribed to the difference in the projectile structure. Preliminary experimental spectra detected in GARFIELD in coincidence with ERs have been compared to the Hybrid Exciton Model predictions. At least in the GARFIELD angular range analyzed up to now, using an initial exciton number estimated by the empirical trend reported in [14], the $\alpha$-particle spectra seem to be reasonably reproduced (except for a small part) at the most forward angles. On the contrary, using the same initial parameters, the fast emission of protons is largely overestimated. A more complete analysis is needed, and is in progress, to understand the fast emission process. The detector performances in terms of mass identification and energy resolutions reached in the present experiment will allow to study all the different light-charged particles decay channels. Moreover, the larger angular range in which the particles have been identified, with respect to older experiments, will permit to better disentangle and study the pre-equilibrium emission. Multiplicities, angular distributions and exclusive $\alpha-\alpha, \alpha-\mathrm{N}$ correlations will be also extracted. Information on the more peripheral collisions like the decay of the projectile (break-up) have also been collected and will be studied in the future. For 
example, the complete reconstruction of the projectilelike decay in the two systems could be an extra observable of their $\alpha$-structure. From the theoretical point of view the Hybrid Exciton Model calculation has shown the need to be upgraded in order to solve he proton-alpha discrepancy and include all the possible decay channels. The use of codes implementing other theoretical approaches in also in the road-map; among the other the dynamical AMD code described in [18] has recently been considered.

\section{References}

[1] L.R. Hafstad, E. Teller, Phys. Rev. 54 (1938)681

[2] W. Von Oertzen et al., Phys. Rep. 432 (2006) 43-113.

[3] K. Ikeda et al., Prog. Theor. Phys. Suppl 464 (1968)

[4] H. Feldmeier et al., Nucl. Phys. A 586, 493(1995)

[5] T. Neff, H. Feldmeier, R. Roth, Nucl. Phys. A 752, 94(2005)

[6] H. Horiuchi, Y. Kanada-En'Yo, Nucl. Phys. A 612, 394c (1997)

[7] Y. Kanada-EnYo, H. Horiuchi, Prog. Th. Phys. Suppl. 142 (2001) 206

[8] SPES website https://web.infn.it/spes/

[9] ISOLDE website https://hie-isolde.web.cern.ch/

[10] SPIRAL2 website http://www.ganil-spiral2.eu/spiral2

[11] O.V. Fotina et al. Int. Journ. Mod. Physics E 19 (2010) 1134

[12] J.J. Griffin , Phys. Rev. Lett. 17 (1966) 478

[13] M. Blann, M.B. Chadwick, Phys. Rev. C 62 (2000) 034604

[14] N. Cindro et al., Phys. Rev. Lett. 66 (1991) 868

[15] O. Fotina et al., EPJ Web of Conferences 66, 03028 (2014)

[16] J. Cabrera et al. Phys Rev. C 68 (2003) 034613

[17] M. Bruno, F. Gramegna et al., Eur. Phys. J. A49 (2013) 128

[18] A. Ono, EPJ Web of Conf. 3100023 (2012) 